\title{
A Retrospective Case-Control Study, Factors Affecting Treatment Outcomes for Pulmonary Tuberculosis in İstanbul, Turkey
}

\author{
Aylin Babalık' 'Zeki Kılıçaslan², Şule Kızıltaş3 ${ }^{3}$ Serap Gencer ${ }^{4}$, Gül Öngen ${ }^{5}$ \\ ${ }^{1}$ Department of Pulmonology, Süreyyapaşa Chest Diseases and Thoracic Surgery Training and Research Hospital, Istanbul, Turkey \\ ${ }^{2}$ Department of Pulmonology, Istanbul University Istanbul Faculty of Medicine, Istanbul, Turkey \\ ${ }^{3}$ Department of Pulmonology, Kadıköy Tuberculosis Dispensary, Istanbul, Turkey \\ ${ }^{4}$ Department of Infectious Diseases and Clinical Microbiology, Dr. Lütfi Kırdar Kartal Training and Research Hospital, Istanbul, Turkey \\ ${ }^{5}$ Department of Pulmonology, Istanbul University Cerrahpaşa Faculty of Medicine, Istanbul, Turkey, Department of Pulmonology, \\ istanbul, Turkey
}

\begin{abstract}
Background: Tuberculosis is a public health problem and its transmission is a threat to the community.

Aims: The aim of this study was to determine the factors influencing the treatment outcomes and the effectiveness of the National Tuberculosis Program (NTP) in relation to the application of the directly observed treatment, short-course (DOTS) program in various sites in Istanbul, Turkey.

Study Design: Case-Control Study

Methods: A case-control study was used, where cases and controls were randomly selected from the Turkish Tuberculosis National Database, which includes complete data on treatment outcomes for patients recorded in the database from January 1, 2006 to December 31, 2009 and had one year follow-up.

Results: The case group was composed of 464 patients with adverse outcome, while the control group was composed of 441 patients who had been cured of disease. Factors associated with adverse treatment outcome were $>65$ years of age (OR: 3.39 (1.99-5.76)); male gender (OR:2.11 (1.49-2.99)); born outside Turkey (OR: 5.48 (2.13-14.04)); co-morbidity (OR: 1.85 (1.29-2.65)); bilateral radiologic lesions (OR: 2.07 (1.41-3.00); previous treatment history (OR: 3.99 (2.78-5.74)); $3^{\text {rd }}$ month positive microscopy (OR: 4.96 (3.04-8.09)) and any H\&R +/- others multidrug resistant (MDR) resistance (OR: 22.64 (6.92-74.08)). There was no association between the adverse treatment outcome and the application site of direct observation treatment, short course (DOTS) delivery and the supervisors.
\end{abstract}

Conclusion: Our findings indicate similar quality in DOTS application and supervision among patients with or without adverse treatment outcomes. However, patients with certain characteristics should be carefully monitored and aggressively treated.

Key Words: Tuberculosis, treatment outcome, affecting factors

Received: 02.10.2012

Accepted: 23.01.2013

\section{Introduction}

Tuberculosis (TB) is a public health problem and its transmission is a threat to the community. The National Tuberculosis Program (NTP) and its health staff are responsible for ensuring the regular intake of necessary drugs by patients. There is a need for NTPs to establish and maintain systems that maximise patient access to care, and train and supervise health workers to provide patient-centred care in order to facilitate patient adherence. Additionally, it is critical for the NTP to implement two components of the Stop TB Strategy including engaging other care providers and involving communities (1).

The global targets for tuberculosis control, set by the World Health Assembly (WHA) in 1991, had a detection rate of at least $70 \%$ and a cure rate of at least $85 \%$ of new sputum smear-positive TB cases by 2000, which was later revised to
$2005(2,3)$. Reductions in the disease burden achieved to date follow 15 years of intensive efforts at a country level, to implement the directly observed treatment, short-course (DOTS) strategy (1995-2005) and the Stop TB Strategy (launched in 2006) (4). Recent studies suggest that countries achieving the global targets would then see a fall in incidence of $8-12 \%$ per year (cases reduced by $50 \%$ in as little as 6-9 years) and an even faster reduction in mortality of $9-13 \%$ per year $(50 \%$ reduction in 5 years or less) (5).

DOTS has expanded in Turkey since 2006 (4). In 2008, treatment success was achieved in $92 \%$ of smear positive new cases. Istanbul has the highest incidence of tuberculosis in Turkey (44.1 per 100000 population). The prevalence of adverse treatment outcomes using the direct observation of treatment is defined as default, death and low failure $(\sim 8 \%)$. However, it is important to understand modifiable 
reasons for adverse treatment outcomes to improve the success rate (6).

The aim of this study was to determine factors influencing the treatment outcomes and the effectiveness of NTP in relation to the application of the DOTS program in various sites in Istanbul, Turkey.

\section{Material and Methods}

\section{Study Design and Study Populations:}

A total of 905 patients divided into control $(n=441$, cured patients) and case ( $n=464$, patients with an adverse treatment outcome) groups were included in this case-control study based on the random selection of cases and controls from the Turkish Tuberculosis National Database that includes complete data on the treatment outcomes for patients who were recorded in the database from January 1, 2006 to December 31, 2009 and had one year follow-up. The random selection of case and control groups from each centre registered in the database was based on being $\geq 18$ years old, having a diagnosis and treatment of the disease from 2006 to 2009 and one-year follow up within this period. Currently, there were 11,186 patients with smear- and/ or culture-positive pulmonary TB. We estimated power 80 with alpha level 0.05 using two sided test and with 400 cases and 400, Group 1 proportion: 0.45, Group 2 proportion: 0.55. Ethical approval for the study was obtained from the institutional review board of Istanbul University in accordance with the Helsinki recommendations (Approval\# 2011/1543-718).

\section{Data collection}

There are 32 TB dispensaries in Istanbul, and each collaborates with one of two main diagnostic laboratories subject to external quality control from the national TB reference laboratory in Ankara (1). The National Tuberculosis database follows the 'WHO' country report (CISID) and case-based recording data standards (Euro TB data file). The data on treatment outcomes, socio-demographics (age, gender, marital status, education level, smoking history), clinical features (previous treatment history, co-morbid diseases, side effects, radiology lesions, radiologic cavity, $3^{\text {rd }}$ month microscopy variables), application site and responsible person for DOTS were collected from medical records in the database.

\section{TB Patients Treatment and Definition of Treatment Outcome}

New patients with pulmonary TB received a standardised short-course chemotherapy consisting of two months of isoniazide $(H)$, rifampicin $(R)$, pyrazinamide $(Z)$, and ethambutol (E), followed by four months of HR. Previously treated patients received two months of HRZE and streptomycin (S), one month of HRZE, and five months of $\operatorname{HRE}(1,7)$. Patients were followed monthly at TB dispensaries and received TB drugs under DOTS every day at the nearest DOTS treatment facility.

We defined clinical outcomes as follows:

Cure: A patient whose sputum smear or culture was positive at the beginning of the treatment but who was smear- or culture-negative in the last month of treatment and on at least one previous occasion.
Treatment failure: A patient whose sputum smear or culture is positive at 5 months or later during treatment. Also included in this definition are patients found to harbour a multidrug-resistant (MDR) strain at any point of time during the treatment, whether they are smear-negative or -positive.

Died: A patient who dies for any reason during the course of treatment.

Default: A patient whose treatment was interrupted for 2 consecutive months or more (7). Clinical outcome was evaluated 12 months after commencement of treatment. Default, death, and treatment failure outcomes were combined as any adverse outcome.

\section{Case Characteristics and Definition of Exposures}

Cases of TB were classified according to bacteriological results, anatomical site, and history of previous treatment (1, 7). New patients had either never received treatment for TB, or had taken anti-TB drugs for less than 1 month. Previous treatment history was categorised as relapsed cases (a patient previously treated for TB who had been declared cured or treatment completed and were currently diagnosed with positive smear or culture), interrupted treatment (a patient who returns for treatment, positive bacteriologically, following interruption of treatment for two months or more), and treatment failure (a patient who is started on re-treatment regimen after failing previous treatment, i.e., having a positive bacteriological result at month 5 of treatment).

Exposure was considered in terms of gender, age groups $(18-35,36-50,51-65,>65)$, country of birth (Turkey, other county), marital status (married, divorced, single), smoking history (present or absent), education level (illiterate, literate, primary school, high school, university), co-morbidity (any present co-morbidity, absent), side effect (present, absent), radiologic lesions (unilateral or bilateral lesions), radiologic cavity (absent, present), previous treatment history (none, any previous treatment history), $3^{\text {rd }}$ month microbiological positivity and any resistance (none, resistant to any drugs, resistant to INH and RIF +/- other drugs (MDR). The application site of DOTS is defined as TB dispensaries that are part of the National TB Program, Health Care Centres, primary health care outpatient clinics, and other locations, including the patient's house, work place and pharmacy where treatment is supervised by non-TB specialists such as pharmacy practitioners.

\section{Data Handling and Statistical Analysis}

Data from the registry was provided in digital format and analysed using the Statistical Program for the Social Sciences software package, SPSS 16.0 (IBM SPSS; Armonk, New York). We randomly chose 20 cases from the computerised database to check against the original registry and found $100 \%$ concordance for all data elements. Continuous variables were summarised using mean and standard deviation (SD) or median and quartiles. We used Logistic Regression (Enter model) and calculated adjusted all odds ratios (OR), 95\% confidence intervals $(95 \% \mathrm{Cl})$ by three variables known to affect the outcome: age, history of prior treatment, gender. 


\section{Results}

In this study including 905 patients overall, control and case groups were composed of 441 (48.7\%) and 464 (51.3\%) patients, respectively. Overall, $45.0 \%$ of all patients and $51.2 \%$ and $39.0 \%$ of patients in control and case groups, respectively, were in the age group of $18-35$ years. Males comprised $78.5 \%$ of the overall population and $48.4 \%$ of patients were primary school graduates. The majority of patients in the control (98.6\%) and case (95.0\%) groups were born in Turkey. Married patients comprised $52.8 \%$ and $56.7 \%$ of patients, while smokers accounted for $34.5 \%$ and $33.4 \%$ of patients in the control and case groups, respectively. Rate of co-morbidity, other country of birth, bilateral radiology lesions, cavity, history of previous treatment, $3^{\text {rd }}$ microscopy positivity, drug resistance to any anti-TB antibiotic that was tested $(H, R, E, S)$, and $H \& R+/$ - others (MDR) was higher in the case group than in the control group. Healthcare centres were the leading application sites of DOTS in the overall population (45.9\%) and in 47.4 and $45.9 \%$ of patients in the control and case groups, respectively. Most of the supervisors of DOTS were healthcare workers in the overall population (69.4\%), control (69.2\%) and case $(69.4 \%)$ groups (Table 1$)$.

There was a significant positive trend of increasing risk for adverse treatment outcome with age with nearly twoand three-fold increase in odds of an adverse treatment outcome among patients aged 51-65 years and >65 years, respectively. Male gender and the presence of co-morbidity were associated with almost doubled odds for an adverse treatment outcome. Birth outside of Turkey was associated with a more than five-fold increase in odds for adverse treatment outcome. Bilateral radiologic lesions and the presence of a cavity were associated with a doubling of the odds for an adverse treatment outcome. Relapse cases and interrupted treatment in the subgroup of previous treatment was associated with over two-fold and ten-fold increase in odds for adverse treatment outcome, respectively. Positive microscopy in the $3^{\text {rd }}$ month was associated with a five-fold increase in odds for adverse treatment outcome. Any resistance was associated with a four-fold increase in the odds for an adverse treatment outcome. Any $\mathrm{H} \& \mathrm{R}+/$ - others (MDR) resistance was strongly associated with adverse treatment outcome (OR: $20.1995 \% \mathrm{Cl}(6.03$ - 67.62)).

\section{Discussion}

In this study, our hypothesis was to determine the factors influencing the treatment outcomes and the effectiveness of the NTP in relation to the application of the DOTS program in various sites in Istanbul, Turkey. Our findings revealed no differentiation between the application sites of DOTS delivery and DOTS supervisors as to adverse treatment outcomes. An association of the adverse treatment outcome with age, male gender, birth outside Turkey, co-morbidity, bilateral radiologic lesions and cavities, $3^{\text {rd }}$ month microscopy positivity, relapse, interrupted treatment in the subgroup of previous treatment and resistance was evident.
In our study, there was a significant positive trend of increased risk for adverse treatment outcome with age with almost two- and three-fold increase in odds of an adverse treatment outcome among patients aged 51-65 years and $>65$ years, respectively. According to data specific to Turkey, the highest rate of death was reported to be seen for the age group >65 (13.4\%) (6), while a significant association between death and older age was consistently reported in several studies that may be partly explained by the effect of co-morbidity confounders (8-15). Given that discontinuation of treatment has also been observed in a number of other conditions that require prolonged drug therapy, such as cardiovascular disease, rheumatic fever, leprosy, epilepsy, diabetes, and malaria prophylaxis, as well as the self-administration of oral contraceptives (16), co-morbidity was associated with a near doubling of the odds for an adverse treatment outcome in our study population.

Gender differences in tuberculosis epidemiology may arise either as a consequence of differences in biological functioning or due to differences in exposure as a consequence of differences in the societal roles of men and women (17). In our study, male gender was associated with almost doubled odds for an adverse treatment outcome. According to Turkey's statistics, the rate of treatment success for males was lower than for female patients and the M/F treatment success rate reported in 2008 was $89.9 / 92.7 \%$ (6). Likewise, some studies showed that male gender was associated with an increased risk for death among tuberculosis cases (8).

As in past studies, which showed that foreign-born patients were at higher risk for default $(18,19)$, in our study, being born outside Turkey was associated with a more than fivefold increase in odds for adverse treatment outcome.

Success among re-treatment case types is normally lower than that for new patients-more so for treatment-after-failure (since previous failure may have been due to drug resistance) and treatment-after-default cases (since cases that defaulted previously are likely to have poor compliance and/or drug resistance) than for relapse cases (20).

Globally, the treatment success rate for retreatment cases was low (72\%), in comparison to new cases (87\%) (6). According to Turkey's statistics for $2008,75.7-92.3 \%$ of the treatment success rate was found to be lower in previously treated cases, than in new cases (6). In our study, relapse cases and interrupted treatment in a subgroup of previous treatment were associated with a more than two-fold and ten-fold increase in odds for adverse treatment outcome, respectively.

In our study, any resistance doubled while H\&R +/- others (MDR) resistance was associated with a twenty-fold increase in odds for an adverse treatment outcome. According to the statistics for Turkey, the MDR rate was approximately $5 \%$ in all cases. In a past study concerning evaluation of the influence of multidrug resistance on tuberculosis treatment outcomes with standardised regimens, failure rates and relapse rates were reported to be higher in countries where the prevalence of initial multidrug resistance exceeded $3 \%$ than in countries where multi-drug resistance was lower than 3\% (21).

Smears should be converted to negative in the majority of new smear-positive pulmonary TB patients after 2 or 3 months 
Table 1. Adjusted odds ratios with sex, age group and previous treated history of association for adverse outcomes with selected clinical and demographic factors

\begin{tabular}{|c|c|c|c|c|}
\hline Patients Characteristics & Control Group $1 \mathrm{n}(\%)]$ & Case Group ${ }^{2}$ [n (\%)] & Total Patients & Adjusted $\mathrm{OR}^{3}$ \\
\hline Number of cases & $441(100.0)$ & $464(100)$ & $905(100)$ & - \\
\hline \multicolumn{5}{|l|}{ Age group [years] } \\
\hline $18-35$ & $226(51.2)$ & $181(39.0)$ & $407(45.0)$ & $-1.0-$ \\
\hline $36-50$ & $123(27.9)$ & $126(27.2)$ & $249(27.5)$ & $1.01(0.72-1.41)$ \\
\hline $51-65$ & $68(15.4)$ & $98(21.1)$ & $166(18.3)$ & $1.53(1.04-2.25)$ \\
\hline$>65$ & $24(5.4)$ & $59(12.7)$ & $83(9.2)$ & $3.39(1.99-5.76)$ \\
\hline \multicolumn{5}{|l|}{ Gender } \\
\hline Female & $127(28.8)$ & $68(34.9)$ & $195(21.5)$ & $-1.0-$ \\
\hline Male & $314(71.2)$ & $396(55.8)$ & $710(78.5)$ & $2.11(1.49-2.99)$ \\
\hline \multicolumn{5}{|l|}{ Country of Birth } \\
\hline Turkey & $435(98.6)$ & $441(95.0)$ & $876(96.8)$ & $-1.0-$ \\
\hline Other Country & $6(1.4)$ & $23(5.0)$ & $29(3.2)$ & $5.48(2.13-14.04)$ \\
\hline \multicolumn{5}{|l|}{ Marital Status } \\
\hline Married & $233(52.8)$ & $263(56.7)$ & $496(54.8)$ & $-1.0-$ \\
\hline Divorced & $12(2.7)$ & $9(1.9)$ & $21(2.3)$ & $0.98(0.71-1.36)$ \\
\hline Single & $185(42.0)$ & $167(36.0)$ & $352(38.9)$ & $0.43(0.15-1.19)$ \\
\hline Unknown & $11(2.5)$ & $25(5.4)$ & $36(4.0)$ & - \\
\hline \multicolumn{5}{|l|}{ Smoking } \\
\hline Non-smoker & $117(26.5)$ & $88(19.0)$ & $205(22.7)$ & $-1.0-$ \\
\hline Smoker & $152(34.5)$ & $155(33.4)$ & 307 (33.9) & $0.94(0.62-1.42)$ \\
\hline Unknown & $172(39.0)$ & $221(47.6)$ & $393(43.4)$ & - \\
\hline \multicolumn{5}{|l|}{ Education Level } \\
\hline University & $29(6.6)$ & $23(5.0)$ & $52(13.9)$ & $-1.0-$ \\
\hline High School & $71(16.1)$ & $60(12.9)$ & $131(14.5)$ & $1.11(0.55-2.23)$ \\
\hline Intermediate School & $67(15.2)$ & $53(11.4)$ & $120(13.3)$ & $0.87(0.43-1.78)$ \\
\hline Primary School & $202(46.1)$ & $236(50.9)$ & $438(48.4)$ & $1.20(0.64-2.26)$ \\
\hline no illiterate & $17(3.9)$ & $21(4.5)$ & $38(4.2)$ & $1.51(0.59-3.90)$ \\
\hline Unknown & $55(12.5)$ & $71(15.3)$ & $126(13.9)$ & - \\
\hline \multicolumn{5}{|l|}{ Co-morbidity } \\
\hline Absent & $364(82.5)$ & $326(70.3)$ & $690(76.2)$ & $-1.0-$ \\
\hline Present & $77(17.5)$ & $138(29.7)$ & $215(23.8)$ & $1.85(1.29-2.65)$ \\
\hline \multicolumn{5}{|l|}{ Side Effect } \\
\hline Absent & $413(93.7)$ & $426(91.8)$ & 839 (92.7) & $-1.0-$ \\
\hline Present & $28(6.3)$ & $38(8.2)$ & $66(7.3)$ & $0.99(0.56-1.73)$ \\
\hline \multicolumn{5}{|l|}{ Radiology } \\
\hline Unilateral & $241(54.6)$ & $210(54.6)$ & $451(45.3)$ & $-1.0-$ \\
\hline Bilateral & $140(31.7)$ & $210(31.7)$ & $350(45.3)$ & $2.07(1.41-3.00)$ \\
\hline Unknown Value & $60(13.6)$ & $44(9.5)$ & $104(11.5)$ & - \\
\hline \multicolumn{5}{|l|}{ Radiology Cavity } \\
\hline Absent & $200(45.4)$ & $175(37.7)$ & $375(41.4)$ & $-1.0-$ \\
\hline Present & $181(41.0)$ & $245(52.8)$ & $426(47.1)$ & $1.56(1.16-2.11)$ \\
\hline Unknown & $60(13.6)$ & $44(9.5)$ & $104(11.5)$ & - \\
\hline
\end{tabular}




\begin{tabular}{|c|c|c|c|c|}
\hline \multicolumn{5}{|l|}{ Previous treatment } \\
\hline None & $391(88.7)$ & $308(66.4)$ & $699(77.2)$ & $-1.0-$ \\
\hline Any & $50(11.3)$ & $156(33.4)$ & $206(22.8)$ & $3.99(2.78-5.74)$ \\
\hline Relapsed & $45(10.2)$ & 97 (20.9) & $142(115.5)$ & $2.24(1.59-3.15)$ \\
\hline Interrupted & $5(1.1)$ & $48(10.3)$ & $53(5.9)$ & $10.37(4.06-26.49)$ \\
\hline Treatment failure & $0(0.0)$ & $11(2.4)$ & $11(5.9)$ & - \\
\hline \multicolumn{5}{|l|}{$3^{\text {rd }}$ month microscopy } \\
\hline Negative & $281(63.7)$ & $104(22.7)$ & $385(42.5)$ & $-1.0-$ \\
\hline Positive & $39(8.8)$ & $68(14.7)$ & $107(11.8)$ & $4.96(3.04-8.09)$ \\
\hline Not tested & $121(27.4)$ & $292(62.9)$ & $413(45.6)$ & - \\
\hline \multicolumn{5}{|l|}{ Antibiotic ${ }^{4}$ resistance } \\
\hline None & $306(69.4)$ & $267(57.5)$ & $573(63.3)$ & $-1.0-$ \\
\hline Any resistance & $41(9.3)$ & $104(22.4)$ & $145(16.0)$ & $2.61(1.71-3.99)$ \\
\hline$H \& R+/-$ others (MDR) & $3(0.7)$ & $65(14.0)$ & $68(7.5)$ & $22.64(6.92-74.08)$ \\
\hline Not tested & $94(21.3)$ & $93(20.0)$ & $187(20.7)$ & - \\
\hline \multicolumn{5}{|l|}{ DOTS Place } \\
\hline Dispensaries & $73(16.6))$ & $93(20.0)$ & $166(18.3)$ & $-1.0-$ \\
\hline HealthCare Centre & $209(47.4)$ & $206(44.4)$ & $415(45.9)$ & $0.92(0.63-1.36)$ \\
\hline Others & $117(26.5)$ & $132(28.4)$ & $249(27.5)$ & $0.88(0.57-1.36)$ \\
\hline Non-DOTS & $42(9.5)$ & $33(7.1)$ & $75(8.3)$ & $0.69(0.38-1.26)$ \\
\hline \multicolumn{5}{|l|}{ DOTS Apply } \\
\hline Healthcare Worker & $305(69.2)$ & $322(69.4)$ & $627(69.4)$ & $-1.0-$ \\
\hline Others & $94(21.3)$ & $109(23.5)$ & $203(23.5)$ & $0.88(0.61-1.26)$ \\
\hline Non-DOTS & $42(9.5)$ & $33(7.1)$ & $75(7.1))$ & $0.73(0.43-1.23)$ \\
\hline
\end{tabular}

of anti-tuberculosis treatment. Sputum smear conversion after 2 or 3 months of treatment is a good predictor of eventual cure if treatment is completed (22). Horne et al. performed a systemic review and meta-analysis to evaluate the accuracy of a positive sputum smear or culture during treatment with standardised regimen with rifampin in the initial phase for predicting failure or relapse in pulmonary TB. As a result, both culture and smear had low positive predictive value (PPV) ranging from 9 to $18 \%$ in predicting poor outcome. In contrast, negative predictive values (NPV) were high (at least 93\%), indicating a negative sputum test result during any month of treatment makes relapse or failure unlikely (23). In our study, $3^{\text {rd }}$ month microscopy positivity was associated with a five-fold increase in odds for adverse treatment outcome. While no significant relationship was observed in our study population between adverse treatment outcomes and smoking, marital status and educational level, a significant relationship between adverse treatment outcome and educational level and smoking was also reported in the literature $(24,25)$.

A systematic review of six controlled trials comparing DOTS with self-administered therapy concluded that DOTS did not improve outcomes (26). The highest success rates were achieved in programs that used DOTS in the context of a full support package, with components such as incentives and enablers (27). The WHO recommends that supervision may be undertaken at a health facility, in the workplace, in the community or at home. Furthermore, The NTP is responsible for training and monitoring of the non-medical observers and a clearly defined line of accountability of staff. For any chosen method of supervision and administration of treatment, a program must show high sputum smear conversion and cure rates, under routine conditions, in both rural and urban areas. If evaluation shows suboptimal results, the method of supervision and administration of the regimen should be altered and tested in demonstration and training districts (1). DOTS-supervised treatment in Turkey has been provided in TB dispensaries. DOTS supervisors consist of healthcare workers in TB dispensaries, healthcare workers in primary health care centres, healthcare workers in the work place, pharmacists, family members and prison workers (7). In 2010, 97.9\% of TB treatment was carried out under DOTS. While $74.7 \%$ of patients were treated by healthcare workers under DOTS supervision, $25.3 \%$ of patients were treated by family members and other supervisors (6). In our study, most of the DOTS application sites were similarly healthcare centres and most of the DOTS supervisors were healthcare workers, both in the case and 
control groups. There was no significant association between the type of application site and the supervisor of DOTS delivery and the adverse treatment outcome, which indicated the similar quality of DOTS application and supervision.

Nevertheless, high success rates in Turkey have been suggested to be likely due to the expansion of DOTS in 2006 and aggressive case follow-up, as shown by consistent reductions in treatment default by $5.7 \%$ in $2005,4.7 \%$ in $2006,4.0 \%$ in 2007 and $3.2 \%$ in 2008 , and the reduction in adverse treatment outcome by $19.2 \%$ between 1998 and 2000 in Istanbul (28).

Achievement of the global targets depends on the ability of countries to accelerate coverage of the population with DOTS, whilst sustaining high cure rates, and the ability of Tuberculosis programs to increase case detection, through the provision of effective services, social mobilisation, and involvement of the private sector. In this regard, our findings show the compatibility with the increasing success rate of TB treatment outcome in Turkey.

However, retrospective design seems to be the major limitation of the present study, since we could only review DOTS supervisors monitoring records in a retrospective manner. Obviously, to evaluate the DOTS quality, prospective studies are necessary.

In conclusion, our findings indicate the similar quality in DOTS application and supervision among patients with or without adverse treatment outcome. However, patients with certain characteristics compatible with being a significant determinant of adverse treatment outcome, such as older age, co-morbid disorder, extensive radiologic disease, previous treatment history, $3^{\text {rd }}$ month microscopy positivity and drug resistance, should be carefully monitored and aggressively treated.

Ethics Committee Approval: Ethics committee approval was received for this study.

Informed Consent: Written informed consent was obtained from patients who participated in this study.

Peer-review: Externally peer-reviewed.

Author contributions: Concept - A.B.; Design - A.B., Z.K.; Supervision - A.B., Z.K.; Resource - Ş.K., S.G.; Materials - A.B., Ş.K.; Data Collection\&/or Processing - A.B., S..K., S.G.; Analysis\&/or Interpretation - A.B.; Literature Search - A.B., Ş.K.; Writing - A.B., Z.K.; Critical Reviews - A.B., Z.K.

Acknowledgements: The authors thank the American Thoracic Society and Turkish Thoracic Society for their sponsorship of the Methods in Epidemiology, Clinical, and Outcomes Research (MECOR) course and Stephen A. McCurdy, MD MPH who is from MECOR faculty, during which this study was conceived and developed. We also thank the patients and staff involved with the Istanbul TB programme.

Conflict of Interest: No conflict of interest was declared by the authors.

Financial Disclosure: No financial disclosure was declared by the authors.

\section{References}

1. World Health Organization. Treatment of Tuberculosis: Guidelines. 4th ed. WHO/HTM/TB/2009.420. Geneva, Switzerland: WHO, 2009.
2. World Health Organization. Forty-fourth World Health Assembly, resolutions and decisions. Resolution WHA 44.8. WHA44/1991/ REC/1. Geneva, Switzerland: WHO, 1991.

3. Kumaresan J, Heitkamp P, Smith I, Billo N. Global Partnership to Stop TB: a model of an effective public health partnership. Int J Tuberc Lung Dis 2004;8:120-9.

4. World Health Organization. Global Tuberculosis Control: The burden of disease caused by TB. WHO/HTM/TB/2011.16. Geneva, Switzerland: WHO, 2011.

5. Dye C Garnett GP, Sleeman K, Williams BG. Prospects for worldwide tuberculosis control under the WHO DOTS strategy. Lancet 1998;352:1886-91. [CrossRef]

6. Bozkurt H, Türkkanı MH, Musaonbaşıoğlu S, Yıldırım A, Baykal F. Fight against tuberculosis 2011 report of Turkey. Ankara, Ministry of Health of Turkey Republic; 2011.

7. Akdağ R, Tosun N, Erkoç Y, Bozgan T, Bozkurt H. Diagnosis and treatment of tuberculosis. Ankara, Ministry of Health of Turkey Republic; 2011.q

8. Lefebvre N, Falzon D. Risk factors for death among tuberculosis cases: analysis of European surveillance data. Eur Respir J 2008;31:1256-60. [CrossRef]

9. Nájera-Ortiz JC, Sánchez-Pérez HJ, Ochoa-Díaz H, Arana-Cedeño M, Lezama MS, Mateo MM. Demographic, health services and socio-economic factors associated with pulmonary tuberculosis mortality in Los Altos Region of Chiapas, Mexico. Int J Epidemiol 2008;37:786-95.

10. Vasankari T, Holmström P, Ollgren J, Liippo K, Ruutu P. Treatment outcome of extra-pulmonary tuberculosis in Finland: a cohort study. BMC Public Health 2010;10:399. [CrossRef]

11. Farah MG, Tverdal A, Steen TW, Heldal E, Brantsaeter AB, Bjune G. Treatment outcome of new culture positive pulmonary tuberculosis in Norway. BMC Public Health 2005;5:14. [CrossRef]

12. Faustini A, Hall AJ, Perucci CA. Tuberculosis treatment outcomes in Europe: a systematic review. Eur Respir J 2005;26:503-10. [CrossRef]

13. Baussano I, Pivetta E, Vizzini L, Abbona F, Bugiani M. Predicting tuberculosis treatment outcome in a low-incidence area. Int J Tuberc Lung Dis 2008;12:1441-8.

14. Talay F, Kumbetli S, Altin S. Factors associated with treatment success for tuberculosis patients: a single center's experience in Turkey. Jpn J Infect Dis 2008;61:25-30.

15. Vasankari T, Holmström P, Ollgren J, Liippo K, Kokki M, Ruutu P. Risk factors for poor tuberculosis treatment outcome in Finland: a cohort study. BMC Public Health 2007;7:291. [CrossRef]

16. N. Bock. What is the significance of default (treatment interruption) in the treatment of tuberculosis? Toman's Tuberculosis Case detection, treatment, and monitoring questions and answers Edited by T. Frieden

17. Borgdorff MW, Nagelkerke NJ, Dye C, Nunn P. Gender and tuberculosis: a comparison of prevalence surveys with notification data to explore sex differences in case detection. Int $\mathrm{J}$ Tuberc Lung Dis 2000;4:123-32.

18. Kapella BK, Anuwatnonthakate A, Komsakorn S, Moolphate S, Charusuntonsri $\mathrm{P}$, Limsomboon $\mathrm{P}$, et al. Directly observed treatment is associated with reduced default among foreign tuberculosis patients in Thailand. Int J Tuberc Lung Dis 2009;13:232-7.

19. Caylà JA, Rodrigo T, Ruiz-Manzano J, Caminero JA, Vidal R, García JM, et al. Tuberculosis treatment adherence and fatality in Spain. Respir Res 2009;10:121. [CrossRef]

20. Indicators for Program Outcomes. Compendium of Indicators for Monitoring and Evaluating National Tuberculosis Programs WHO/HTM/TB/2004.344

21. Mak A, Thomas A, Del Granado M, Zaleskis R, Mouzafarova N, Menzies D. Influence of multidrug resistance on tuberculosis 
treatment outcomes with standardized regimens. Am J Respir Crit Care Med 2008;178:306-12. [CrossRef]

22. COMPEDIUM of indicators for monitoring and evaluating national tuberculosis programs WHO/HTM/TBT 2004.344

23. Garrido Mda S, Penna ML, Perez-Porcuna TM, de Souza AB, Marreiro Lda $S$, Albuquerque $B C$, et a. Factors associated with tuberculosis treatment default in an endemic area of the brazilian Amazon: a case control-study. PLoS One 2012;7:e39134. [CrossRef]

24. Horne DJ, Royce SE, Gooze L, Narita M, Hopewell PC, Nahid P, et al. Sputum monitoring during tuberculosis treatment for predicting outcome: systematic review and meta-analysis. Lancet Infect Dis 2010;10:387-94. [CrossRef]
25. Lin HH, Ezzati M, Murray M. Tobacco smoke, indoor air pollution and tuberculosis: a systematic review and meta-analysis. PLoS Med 2007;4:e20. [CrossRef]

26. Volmink J, Garner P. Directly observed therapy for treating tuberculosis. Cochrane Database Syst Rev 2007;4:CD003343.

27. Suárez PG, Watt CJ, Alarcón E, Portocarrero J, Zavala D, Canales R, et al. The dynamics of tuberculosis in response to 10 years of intensive control effort in Peru. J Infect Dis 2001;184:473-8. [CrossRef]

28. Kilicaslan Z, Oztürk F, Sarimurat N, Cuhadaroglu C, Caglar E, Erem A. Microscopic examination and treatment outcomes of new pulmonary tuberculosis cases in Istanbul dispensaries between 1998 and 2000. Int J Tuberc Lung Dis 2003;7:1059-63. 